\title{
Individuelle Prognose bei kritisch kranken Patienten mit septischem Schock durch ein neuronales Netz?
}

\author{
E. Hanisch ${ }^{1}$, M. Büssow ${ }^{1}$, R. Brause ${ }^{2}$ und A. Encke ${ }^{1}$ \\ ${ }^{1}$ Klinik für Allgemeinchirurgie (Direktor Prof. Dr. A. Encke), Johann-Wolfgang-Goethe-Universität Frankfurt/Main \\ ${ }^{2}$ Institut für Neuroinformatik und Parallele Systeme, Johann-Wolfgang-Goethe-Universität Frankfurt/Main
}

\section{Neural networks as a prognostic tool in critically ill patients}

Summary. From 1.11. 93 to 30. 3. 97, 1149 patients were prospectively studied during their ICU stay. Of them,114 met the criteria of septic shock, with lethality of $47.3 \%$. A neural network was trained with datasets from 91 of these 114 patients. Testing the trained neural network with the remaining 23 patients, the following result was obtained: all 10 patients dying from septic shock were correctly predicted; of 13 surviving patients, 12 were correctly identified (sensitivity $100 \%$; specifity $92.3 \%)$.

Key words: Neural network - Critically ill patients.

Zusammenfassung. Im Zeitraum 1. 11. 1993 bis 30. 3 . 1997 wurden 1149 allgemeinchirurgische Intensivpatienten prospektiv erfaßt, von denen 114 die Kriterien des septischen Schocks erfüllten. Die Letalität der Patienten mit einem septischen Schock betrug 47,3\%. Nach Training eines neuronalen Netzes mit 91 (von insgesamt $n=114$ ) Patienten ergab die Testung bei den verbleibenden 23 Patienten bei der Berücksichtigung von Parameterveränderungen vom 1. auf den 2. Tag des septischen Schocks folgendes Ergebnis: Alle 10 verstorbenen Patienten wurden korrekt als nicht überlebend vorhergesagt, von den 13 Überlebenden wurden 12 korrekt als überlebend vorhergesagt (Sensitivität $100 \%$; Spezifität 92,3\%).

Schlüsselwörter: Neuronales Netzwerk - Prognose - Intensivpatient.

In der Intensivmedizin wird die Letalität der Patienten hauptsächlich davon bestimmt, ob es zur Ausbildung eines Multiorganversagens („multiple organ failure“, MOF) kommt und wie schwer dieses ausgeprägt ist [7, 17, 29]. Nach einer Studie von Knaus et al., in der 2719 Patienten mit Versagen eines oder mehrerer Or- gansysteme untersucht wurden, ergab sich eine Letalität von $22 \%$ für Patienten, bei denen nur an einem einzigen Tag das Versagen eines einzelnen Organsystems registriert wurde. Wenn 2 oder gar 3 Organsysteme betroffen waren, stieg die Sterblichkeitsrate auf 52 bzw. $80 \%$ an [22].

Zum Zweck der Verlaufsdokumentation und gerade auch zur prognostischen Einschätzung in der frühen Behandlungsphase wurden verschiedene Scoringsysteme entwickelt. Erwähnt seien das APACHE-II-System (,acute physiology and chronic health evaluation system II“) [23, 24, 26]), der MOF-Score [18], der „Sepsisscore von Elebute und Stoner“ [19], der „MannheimPeritonitis-Index“ (MPI) [30] und der ,injury severity score“" (ISS) [2].

Die vorliegende Problematik, Verläufe von verschiedenen Variablen eines Patienten $\mathrm{zu}$ beurteilen und die zukünftige Entwicklung vorherzusagen, entspricht der allgemeinen Problematik einer Zeitreihenanalyse [6].

Ein wichtiger, methodisch relevanter Bereich für eine Zeitreihenanalyse ist z. B. die Vorhersage von Aktien- und Wechselkursen mit Hilfe verschiedener Variablen wie Aktienindex, Dollarkurs und anderen Wirtschaftszahlen. Im Unterschied zu konventionellen Methoden wie Fourrier-Analyse oder gleitenden Mittelwerten beruhen diese Erfolge auf dem Einsatz von adaptiven Systemen, speziell künstlicher neuronaler Netze. Dazu wird ein Netz mit den bekannten Daten trainiert, bis es nicht nur in der Vergangenheit die richtige, bekannte Vorhersage macht, sondern auch bei aktuellen Werten eine ausreichende Genauigkeit erreicht. Die dazu verwendeten Netze lassen sich als Approximationsnetze bezeichnen, da sie als Aufgabe haben, für einen Satz von vorgegebenen Parametern den dazugehörigen Funktionswert einer unbekannten Funktion möglichst gut zu approximieren. Diese Technik der neuronalen Netze wird in vorliegender Arbeit für die Prognoseabschätzung chirurgischer Intensivpatienten eingesetzt. 


\section{Methodik}

Neuronale Netze werden auch als ,,artificial neural networks“ bezeichnet; es sind informationsverarbeitende Systeme, die aus einer Vielzahl einfacher „Zellen“ bestehen und sich Informationen in Form der Aktivierung der Zellen über gerichtete Verbindungen zusenden (Analogie zu Aktionspotentialen bei ,richtigen“ Neuronen).

Die Grundelemente oder Neuronen sind (in der Regel) in Schichten organisiert, wobei jedes Neuron mit den Neuronen der nachgeschalteten Schichten verbunden ist.

Die verschiedenen Netze unterscheiden sich, je nach Einsatzgebiet in verschiedenen Aspekten, verschiedene Eigenschaften sind aber bei allen heute gebräuchlichen neuronalen Netzen zu finden:

- neuronale Netze „lernen“ durch Erfahrung;

- sie sind insbesondere gut verwendbar in Bereichen, wo die konventionelle Statistik versagt: bei komplexen, nicht-linearen Problemen;

- die zur Erreichung optimaler Ergebnisse notwendige Fallzahl hängt von der Art des zu lösenden Problems und der Zahl der Eingangsvariablen $\mathrm{ab}$.

Die heute gebräuchlichsten Typen neuronaler Netze sind das „multilayer-perceptron“, die „radial-base-function“ und das Kohonennetzwerk. In unserer Auswertung wurden die ersten beiden, also das „multilayer-perceptron“ und die „radial-base-function“ verwendet.

Wie schon weiter ober erwähnt, sind neuronale Netze besonders gut geeignet für komplexe, nicht-lineare Probleme.

Während in der konventionellen Statistik die Anforderungen an die auszuwertenden Daten sehr hoch sind (es muß lineare $\mathrm{Zu}$ sammenhänge geben und Stichproben müssen in der Regel standardnormalverteilt sein, was in der Medizin fast nie vorkommt), sind neuronale Netze hier wesentlich anspruchsloser: Außer der Repräsentativität der Daten gibt es kaum weitere Anforderungen.

Wenn man nun ein neuronales Netz zu einem bestimmten Zweck erstellen will, so benötigt man hierfür die in Abb. 1 aufgeführten Schritte.

Bei der Vorbereitung der Daten gilt es, die benötigten Fallzahlen zu berücksichtigen. Diese hängen von der Zahl der relevanten Eingangsvariablen und der Zahl der möglichen Ergebnisklassen (also z.B. Überleben/Nichtüberleben) ab. Als Faustregel kann man von folgenden Zahlen ausgehen:

- Die Anzahl der Datensätze sollte 10mal der Anzahl der Eingangsgewichte der Neuronen (Anzahl der Eingangsvariablen) entsprechen.

- Die Anzahl der Trainingsdatensätze sollte bei $n$ Eingangsvariablen und $\mathrm{m}$ Ergebnisklassen wenigstens $5 \mathrm{x}(n+\mathrm{m})$ betragen.

Auch muß man darauf achten, daß die Häufigkeitsverteilung der Datensätze in bezug auf die Ergebnisklassen nicht zu ,schief“ ist, d.h. wenn es 2 mögliche Ergebnisse gibt (z. B. Überleben/ Nichtüberleben oder männlich/weiblich) sollten auch etwa gleich viele Datensätze dieser Kategorien verwendet werden. Ansonsten besteht die Gefahr, daß das neuronale Netz nicht die Muster der individuellen Gruppen lernt, sondern lediglich die Häufigkeitsverteilung der beiden Gruppen zueinander. Bei mehr Ergebnismöglichkeiten wird entsprechend verfahren.

Eine schiefe Verteilung kann falls nötig z. B. dadurch korrigiert werden, daß man überzählige Fälle der einen Kategorie wegläßt oder, besser, indem man Fälle der Kategorie in der Unterzahl solange kopiert, bis ein ausgeglichenes Ergebnis erzielt wird. Es erhöht sich hiermit zwar nicht die Modellgüte für die Gruppe in der Minderzahl, es lassen sich aber mehr Informationen aus den anderen Gruppen verarbeiten, was besonders bei kleinen Datenbeständen nützlich ist. $\mathrm{Zu}$ einer Verfälschung des Modells kommt es hierbei aber nicht (in der konventionellen Statistik wäre dies der Fall).

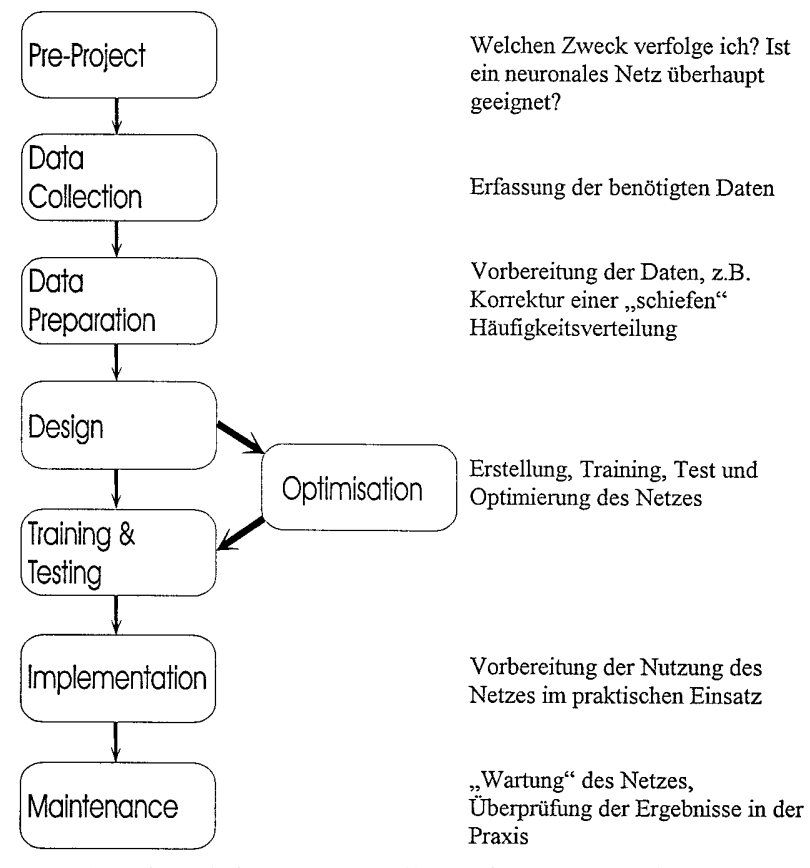

Abb.1. Die Schritte zur Erstellung eines neuronalen Netzes

Ein weiteres Problem, das berücksichtigt werden muß, ist die Gefahr des „Übertrainings“. Ein Übertraining des neuronalen Netzes würde bedeuten, daß es zwar die Daten, mit denen es trainiert wurde, von den Ergebnissen her exakt abbildet, es aber, wenn es die Ergebnisse anderer Datensätze vorhersagen soll, völlig versagt. In diesem Fall wäre dem Netz die Fähigkeit zur Generalisierung des Gelernten, also die Übertragung auf bis dahin unbekannte Fälle verloren gegangen.

Eine Ursache des Übertrainings kann eine zu hohe Zahl von Datensätzen oder Eingangsvariablen sein, eine andere eine ungünstige Verteilung von Trainings-, Validierungs- und Testdatensätzen.

Man unterteilt, um dies zu verhindern und um eine Kontrolle über die Vorhersagegenauigkeit des neuronalen Netzes zu haben, die vorhandenen Datensätze in 3 Gruppen:

Trainingsdatensätze. Sie werden für das Training des Netzes verwendet.

Validierungsdatensätze. Sie dienen der Überprüfung des gelernten; sind die Vorhersageergebnisse mit diesen Datensätzen nicht gut genug, folgt eine weitere Trainingsphase.

Testdatensätze. Sie werden dem neuronalen Netz während der Trainings- und Validierungsphase vorenthalten (sog. „Holdouts“) und dann nach Abschluß des Lernprozesses verwendet, um zu prüfen, wie gut das Netz bis dahin unbekannte Fälle, von denen wir die Ergebnisse aber schon wissen, vorhersagen kann. Es wird hier also die Fähigkeit des Netzes zur Generalisierung des Gelernten geprüft.

Die Einteilung der Datensätze in die 3 oben genannten Gruppen erfolgt normalerweise nach dem Schema 50: 25: 25, d.h. $50 \%$ Trainingsdatensätze, $25 \%$ Validierungsdatensätze usw. Gelegentlich kann es sinnvoll sein, von diesem Schema abzuweichen, z. B. bei sehr großen oder sehr kleinen Datenbeständen. Die Zuordnung der einzelnen Fälle zu den 3 Gruppen sollte nach dem $\mathrm{Zu}-$ fallsprinzip erfolgen; gelegentlich kann die individuelle Zuordnung großen Einfluß auf die Güte der Ergebnisse haben, deshalb sollte man in der Optimierungsphase des Netzes mit mehreren Zuordnungen experimentieren, bei welcher man die besten Ergebnisse erhält. 
Auch die Art des verwendeten Netzes, ob „multilayer-perceptron, backpropagation, feed-forward" oder „radial-base-function“ kann auf die Ergebnisse erhebliche Auswirkungen haben. Generelle Empfehlungen, welche Typen für welche Probleme am besten geeignet sind, kann man aber nicht geben. Dies muß man für jeden speziellen Fall testen.

\section{Patienten}

In einer prospektiven Studie werden seit 1.11.1993 alle allgemeinchirurgischen Intensivpatienten des Klinikums der Johann-Wolfgang-Goethe-Universität Frankfurt/Main erfaßt. Hierbei werden pro Tag und Patient bis zu 245 Parameter aus folgenden Kategorien computergestützt dokumentiert:

- Aufnahmedaten,

- Anamnese,

- Diagnose(n),

- Operation und Narkose,

- Therapeutischer und pflegerischer Aufwand,

- Beatmungsparameter und Blutgasanalysen,

- Klinische Befunde und Vitalparameter,

- Mikrobiologische Befunde,

- Klinische Chemie,

- Pharmakotherapie,

- Ernährung (enteral/parenteral),

- Bilanz und CVVH/Dialyse.

Zur Erfassung der Daten wurde das Datenbankprogramm „Starbase" unter MS Windows 3.11 bzw. Windows '95 verwendet. Die Speicherung der Daten erfolgte in 12 dBase-IV-Datenbankdateien, entsprechend den oben genannten Kategorien. Als Computer wurde ein Notebook mit Intel-486-Prozessor und 8 MB RAM verwendet.

Die Auswertung der Daten wurde im wesentlichen mit dem Tabellenkalkulationsprogramm MS Excel 7.0 vorgenommen. Zur Konstruktion des neuronalen Netzes wurde das Programm „Neural Connection“ (SPSS, München) verwendet.

\section{Definitionen}

Nach der Consensuskonferenz „American College of Chest Physicians 1992" [1]:

„Systemic inflammatory response syndrome“ (SIRS)

SIRS manifestiert sich mit 2 oder mehr der folgenden Bedingungen:

- Temperatur $>38^{\circ} \mathrm{C}$ oder $<36^{\circ} \mathrm{C}$

- Herzfrequenz $>90 / \mathrm{min}$

- Atemfrequenz $>20 /$ min oder $\operatorname{paCO}_{2}<32$ Torr $(<4,3 \mathrm{kpa})$

- Leukocyten $>12000,<4000$ oder $>10 \%$ unreife Formen

Sepsis

Die systemische Antwort auf eine Infektion. Diese ist mit 2 oder mehr der folgenden Bedingungen als ein Ergebnis einer Infektion definiert:

- Temperatur $>38^{\circ} \mathrm{C}$ oder $<36^{\circ} \mathrm{C}$,

- Herzfrequenz $>90 / \mathrm{min}$,

- Atemfrequenz $>20 /$ min oder $\mathrm{paCO}_{2}<32$ Torr $(<4,3 \mathrm{kpa}$ ),

- Leukocyten $>12000,<4000$ oder $>10 \%$ unreife Formen.

Septischer Schock

Sepsis mit Hypotension trotz adäquatem Flüssigkeitsersatz zusammen mit Perfusionsstörungen, die eine Lactatacidose, eine Olig- urie oder eine akute Veränderung des mentalen Status miteinschließen sowie der Einsatz von inotropen oder vasopressorischen Medikamenten zur Korrektur einer Hypotension.

\section{Infektion}

Mikrobielles Phänomen, das durch eine inflammatorische Antwort auf die Gegenwart von Mikroorganismen oder durch die Invasion normalerweise sterilen Gewebes durch diese Organismen charakterisiert ist.

\section{Hypotension}

Ein systolischer Blutdruck von $<90 \mathrm{mmHg}$ oder eine Reduktion von mehr als $40 \mathrm{mmHg}$ vom Ausgangswert in Abwesenheit anderer Ursachen für die Hypotension.

\section{Konstruktion des neuronalen Netzes}

Nach Analyse der epidemiologischen Daten der Patienten im Zeitraum 1. 11. 1993-30. 10. 1995 (Tabelle 1) wurde ein neuronales Netz (,neural connection“; SPSS, München) mit folgenden Parametern konstruiert: Geschlecht, Alter, Atemfrequenz, $\mathrm{FiO}_{2}, \mathrm{pO}_{2}$, $\mathrm{pCO}_{2}, \mathrm{pH}$, maximaler Beatmungsdruck, Leukocyten, TPZ, Thrombocyten, AT III, Lactat, Kreatinin, Puls, systolischer RR, Temperatur, Dopamin, Dobutrex, Natriumbicarbonat, CVVH.

Dabei fanden in einem ersten Schritt nur Datensätze von Patienten am ersten Tag des septischen Schocks Eingang in die Berechnungen.

In einem weiteren Schritt wurden die Veränderungen der Parameter vom 1. auf den 2. Tag des septischen Schocks für die Konstruktion des neuronalen Netzes verwendet.

\section{Statistik}

Der $\chi^{2}$ - und U-Test nach Wilcoxon, Mann und Whitney $\left({ }^{\mathrm{x}} p<0,05\right)$ und die Sensitivität und Spezifität werden nach bekannten Formeln berechnet.

\section{Ergebnisse}

Im Zeitraum vom 1. 11. 1993-30. 10. 1995 entwickelten 235 Patienten (43,6\%) kein SIRS; 2 Patienten verstarben in dieser Gruppe, 231 Patienten (42,9\%) entwickelten ein SIRS, 12 Patienten verstarben in dieser Gruppe $(5,2 \%), 73$ Patienten entwickelten eine Sepsis, davon 52 Patienten einen septischen Schock mit einer Letalität von $50 \%$. Diese Patienten sind in Tabelle 1 detailliert analysiert. Der APACHE-II-Wert betrug in der Gruppe septischer Schock-Überlebender 23,8 (11-38), bei den Nichtüberlebenden 25,7 (19-33) am 1. Tag des Intensivaufenthalts und ist damit nicht signifikant unterschiedlich.

Von 1149 Intensivpatienten, die im Zeitraum 1. 11. 1993-30. 3. 1997 erfaßt wurden, erfüllten $n=114$ das Kriterium septischer Schock mit einer Letalität von $n=54$ (47,3\%). Nach Training des Netzes mit 91 Patienten ergab die Testung bei den verbleibenden 23 Patienten eine 93,4\%ige richtige Vorhersage. Bei der Berücksichtigung der Veränderungen vom 1. Tag auf den 2. Tag des septischen Schocks gab das neuronale Netz wiederum nach Training mit 91 Patienten bei den verbleibenden 23 Testpatienten folgende Prognose ab: alle 
Tabelle 1. Patienten mit septischem Schock; Daten als Mittelwert und Bereich (Zeitraum 1.11.1993-30.10. 1995)



${ }^{\mathrm{a}} \mathrm{p}<0,05$

10 verstorbenen Patienten wurden korrekt als nicht überlebend vorhergesagt, von den 13 Überlebenden wurden 12 korrekt als überlebend vorhergesagt (Sensitivität $100 \%$; Spezifität 92,3\%).

\section{Diskussion}

Scoringsysteme werden in der Intensivmedizin häufig eingesetzt [16, 27, 31]. Die z.Z. verwendeten Scoringsysteme, um das Überleben von Patienten auf Intensivstationen vorauszusagen, sind hochspezifisch, d.h. sie sagen das Überleben in ca. $90 \%$ voraus, sind aber nicht sehr sensitiv, d.h. sie sind nur in ca. 50-70\% genau, um den Tod vorauszusagen. Aus diesem Grund gilt die allgemeine Empfehlung, daß diese angesprochenen Scoringsysteme nur für Patientengruppen verwendet wer- den sollten, für den individuellen Patienten sind sie nicht geeignet [28].

Den angesprochenen Scoringsystemen ist gemeinsam, daß alle eingesetzten Variablen als unabhängige Ereignisse behandelt werden. Dadurch können wertvolle Vorhersageinformationen verlorengehen. Durch Einsatz der logistischen Regression hat man versucht, diesem kritischen Punkt gerecht zu werden, es muß jedoch konstatiert werden, daß die sehr komplexe Situation, wie sie für den Intensivpatienten gilt, dadurch nicht erfaßt werden kann. Gerade hierfür eignen sich neuronale Netzwerke besonders, weil sie das ,chaotische Benehmen" biologischer Prozesse modellhaft wiedergeben können [4].

Inzwischen werden neuronale Netzwerke auf einer Reihe von klinischen Gebieten eingesetzt: Appendicitis [13], Herzinfarkt [3], in der Analyse von EEG und EKG 
$[5,9,10,14,21,25]$, daneben in der Lebertransplantation [11] und der Herzklappenchirurgie [20].

Für die Intensivmedizin wurde kürzlich eine Studie vorgestellt, bei der bei septischen Schockpatienten die Performance eines neuronalen Netzwerks mit der eines logistischen Regressionsmodells verglichen worden ist [12]. Dabei schnitt das neuronale Netzwerk entscheidend besser ab. Kritisch zu bewerten ist an dieser Studie, daß zufälligerweise ein einziger Tag des Intensivaufenthalts ausgesucht worden war, ohne diesen Tag näher zu spezifizieren. Dies bedeutet praktisch, daß sowohl Tage in der Frühphase wie auch in der Spätphase des Intensivaufenthalts Verwendung gefunden haben. Für die klinische Situation ist dieses neuronale Netz unbrauchbar. Notwendig ist ein neuronales Netz am Anfang des kritischen Verlaufs. Dieser Forderung wird vorliegende Arbeit gerecht, indem bei septischen Schockpatienten ausschließlich Parameter des 1. Tages bzw. Veränderungen vom 1. auf den 2. Tag nach Diagnosestellung ,septischer Schock" Verwendung für das neuronale Netz finden. Dabei sind Sensitivität und Spezifität sehr hoch, dies vor dem Hintergrund des Versagens des APACHE-II-Score, der bei überlebenden und nicht-überlebenden Patienten der Gruppe septischer Schock identisch ist.

Eine weitere Entwicklung des neuronalen Netzes unter Einschluß der Dynamik des septischen Prozesses könnte eine individuelle Prognosestellung möglich machen, mit dem Ziel, zum frühestmöglichen Zeitpunkt auf die Gefahr eines letalen Verlaufs hinzuweisen und dem mit aller Macht entgegenzusteuern.

Wir möchten abschließend darauf hinweisen, daß der Information des neuronalen Netzes eine unterstützende Funktion zukommt, die den Arzt in seiner Therapiefreiheit nicht einschränkt. Auf gar keinen Fall interpretieren wir den Einsatz des neuronalen Netzes im fatalistischen Sinne, wie dies gegenwärtig für ein Prognosesystem (Stichwort „Todescomputer") geschieht.

Natürlich ist der Einsatz einer „Maschine“, die Informationen über Leben und Tod gibt, mit großen Emotionen belastet, wir gehen jedoch davon aus, daß, wenn es gelingt, die Datenbasis auf mehrere Zentren zu erweitern, um so der Heterogenität des Krankheitsbildes Sepsis gerecht zu werden [15], es möglich sein wird, zu einem sehr frühen Zeitpunkt Warnhinweise zu erhalten, die es erlauben, durch rechtzeitiges therapeutisches Intervenieren die weltweit immer noch sehr hohe Letalität des septischen Schocks zu senken. In diesem Zusammenhang möchten wir noch die Meinung des Philosophen Churchland anführen: „.. . und künstliche neuronale Netze werden uns bald Diagnosen ermöglichen, die viel verläßlicher, schneller und logischer sind, als Menschen es jemals sein können. Derartige Netzwerke können in Zukunft auch rascher und umsichtiger Therapievorschläge ausarbeiten als jeder Arzt“" [8].

\section{Literatur}

1. American College of Chest Physicians - Society of Critical Care Medicine Consensus Conference (1992) Definitions für sepsis and organ failure and guidelines for the use of innovative therapies in sepsis. Crit Care Med 20: 864
2. Baker SP, O'Neill B, Haddon W, Long WB (1974) The injury severity score: A method for describing patients with multiple injuries and evaluation emergency care. J Trauma 14: 187

3. Baxt WG (1991) Use of an artificial neural network for the diagnosis of myocardial infarction. Ann Intern Med 115: 843

4. Baxt WG (1994) Complexity, chaos and human physiology: the justification for non-linear neural computational analysis. Cancer Let 77: 85

5. Bortolan G, Willems JL (1993) Diagnostic ECG classification based on neural networks. J Electrocardiol 26: 75

6. Brause R (1995) Neuronale Netze. Teubner, Stuttgart

7. Caricco CJ, Meakins JL, Marshall JC, Fry D, Maier RV (1986) Multipleorgan-failure syndrome. Arch Surg 121: 196

8. Churchland P (1997) Die Seelenmaschine. Spektrum, Heidelberg

9. Clayton RH, Murray A, Campbell RW (1994) Recognition of ventricular fibrillation using neural networks. Med Biol Eng Comput 32: 217

10. Devine B, Macfarlane PW (1993) Detection of electrocardiographic ,left ventricular strain“ using neural nets. Med Biol Eng Comput 31: 343

11. Doyle HR, Dvorschik I, Mitchell S, Marino S, et al (1994) Predicting outcome after liver transplantation: a connectionist approach. Ann Surg 219: 408

12. Dybowski R, Weller P, Chang R, Grant V (1996) Prediction of outcome in critically ill patients using artificial neural networks synthesised by genetic algorithm. Lancet 347: 1146

13. Eberhard RC, Dobbins RW, Hutton LV (1991) Neural Network paradigm comparisons for appendicitis diagnosis. Proceedings of the fourth annual IEEE Symposium on Computer-based Medical Systems, p 298

14. Edenbrandt L, Heden B, Pahlm O (1993) Neural networks for analysis of ECG complexes. J Electrocardiol 26: 74

15. Encke A, Hanisch E, Lorenz W (1997) Evaluationsmodelle zur Therapieplanung/Standardisierung am Beispiel Sepsis. Langenbecks Arch Chir (im Druck)

16. Foitzik Th, Holle R, Schall R, Moesta Th, et al (1995) Der Heidelberger Wachstation-Score. Chirurg 66: 513

17. Goris RJA, Boekholtz WKF, van Bebber IPT, Nuytinck JKS, Schillings PHM (1986) Multiple-organ failure and sepsis without bacteremia. Arch Surg 121:897

18. Goris RJA, te Boekhorst TPA, Nuytinck JKS, Gimrere JSF (1985) Multiple organ failure - generalized autodestructive inflammation? Arch Surg 120: 1109

19. Grundmann R, Kipping N, Wesoly C (1988) Der „Sepsisscore“ von Elebute und Stoner zur Definition der postoperativen Sepsis auf der Intensivstation. Intensivmedizin 25: 268

20. Katz AS, Katz S, Wickham E, Quijano RC, et al (1993) Prediction of valve-related complications for artificial heart valves using adaptive neural networks: a preliminary study. J Heart Valve Dis 2: 504

21. Kloppel B (1994) Application of neural networks for EEG analysis. Consideration and first results. Neuropsychobiology 29:39

22. Knaus WA, Draper EA, Wagner DP, Zimmermann JE (1985) Prognosis in acute organ-system failure. Ann Surg 202: 685

23. Knaus WA, Draper EA, Wagner DP, Zimmermann JE (1985) APACHE II: A severiy of disease classification system. Crit Care Med 13: 818

24. Knaus WA (1988) The science of prediction and its implications for the clinician today. Theor Surg 3: 93

25. Masic N, Pfurtscheller G (1993) Neural network based classification of single trial EEG data. Artif Intell Med 5: 503

26. Murray B, Fakhry SM, Cooney R, Rutledge R, Meyer AA (1992) Comparison of ICU nursis clinical judgement and APACHE II score in predicting ICU outcome in critical ill surgical patients. Crit Care Med 20: 57

27. Ohmann C, Grofs-Weege W (1992) Scoring-Systeme auf der chirurgischen Intensivstation. Chirurg 63: 1021

28. Suter P, Armaganidis A, Beaufils F, Bonfill X, et al (1994) Predicting outcome in ICU patients. Intensive Care Med 20: 390

29. Tilney NL, Bailey GL, Morgan AP (1973) Sequential system failure after rupture of abdominal aortic aneurysms: An unsolved problem in postoperative care. Ann Surg 108: 117

30. Wacha H, Lindner MM, Feldmann U, Wesch G, et al (1987) Mannheim peritonitis index - prediction of risk of death from peritonitis: Construction of a statistical and validation of an empirically based index. Theor Surg 1: 169

31. Wahl W, Pelletier K, Schmidtmann S, Junginger Th (1996) Erfahrungen mit verschiedenen Scores zur Beurteilung der Prognose bei postoperativen Intensivtherapiepatienten. Chirurg 67: 710

Priv.-Doz. Dr. Dr. E. Hanisch

Klinik für Allgemeinchirurgie

Johann-Wolfgang-Goethe-Universität

Theodor-Stern-Kai 7

D-60590 Frankfurt/Main 